\title{
CDISC SDTM Yes No Unknown or Not Applicable Response Terminology
}

National Cancer Institute

\section{Source}

National Cancer Institute. CDISC SDTM Yes No Unknown or Not Applicable Response

Terminology. NCI Thesaurus. Code C66742.

Terminology codelist used with Yes No Unknown or Not Applicable Response within the Clinical Data Interchange Standards Consortium Study Data Tabulation Model. 